\section{SOBREPESO, UMA NOVA REALIDADE NO ESTADO NUTRICIONAL DE PRÉ-ESCOLARES DE NATAL (RN): EPIDEMIA OU PÂNICO MORAL?}

A interseção da medicina com a moralidade vem de longa data. Nietzsche, filósofo alemão do século XIX, de certo modo, apontava nesta direção em seus escritos sobre a "grande saúde", em "A Gaia Ciência"!: a moral do dever é, freqüentemente, inumana. Atualmente, não diferente, alguns especialistas têm, sem comedimento, comparado os comportamentos relacionados à saúde com os pecados ou crimes, e os tratamentos como algo que pode remover a culpa ${ }^{2}$. Lupton ${ }^{3}$ lembra que os discursos e práticas do conhecimento biomédico têm servido para constituir e regular fenômenos como "normalidade" e "risco" e, exatamente como um sistema moral, distinguido os "bons" e "maus" pacientes.

Não à toa, Campos et al. ${ }^{4}$ trouxeram à tona a idéia de "pânico moral" para designar o discurso estridente que se faz em torno da obesidade, sem que, no entanto, se esmiúce a fragilidade das medidas de obesidade, os interesses em jogo e as incertezas em relação às associações com as doenças.

Não se debate nos estudos, por exemplo, as arbitrariedades dos pontos de corte para classificação. Tampouco têm sido discutidas as implicações que tais discursos podem gerar, legitimando desigualdades em saúde, vez que o aforismo sentencia que depende do indivíduo fazer escolhas corretas, quando, parecem ser escolhas possíveis, ou ainda porque ajuda a estigmatizar os sujeitos. Além disto, pode contribuir para desordens alimentares e distúrbios relacionados à imagem corporal ${ }^{5}$.

Neste sentido, o estudo de Barreto et al. ${ }^{6}$ e o editorial de Escobar et al. ${ }^{7}$ merecem reflexões mais aprofundadas. Um primeiro aspecto pode ser questionado ao se determinar, arbitrariamente, que o "risco de sobrepeso" ocorre para índice de massa corpórea (IMC) com percentil igual ou superior a 85 e "sobrepeso" quando o IMC for igual ou superior ao percentil 95. Ora, o fato de determinado grupo se encontrar nos percentis mais elevados não significa que estejam necessariamente com sobrepeso, apenas que, comparado a outro grupo com tendência diferente, apresentam um maior contingente de pessoas com valores mais elevados. Tome-se como exemplo o fato de uma amostra ter todos os seus sujeitos com "baixo peso", ainda, assim, ter-se-ia parte deste grupo classificado como "excesso de peso", vez que estes se encontrariam com IMC igual ou superior ao percentil 85.

Outro ponto que reflete a arbitrariedade destas investigações diz respeito a uma própria ponderação dos autores "A prevalência encontrada neste estudo pode ser considerada alta, comparada àquelas de inquéritos nacionais anteriores (3\% - 4\%), apesar destes utilizarem definições de sobrepeso diferentes (p. 313, grifo nosso)". Merece destaque o fato de os pesquisadores estarem utilizando definições distintas e, ainda assim, concluindo sobre um possível aumento dos casos de sobrepeso.

O mesmo pode ser aplicado à idéia errônea de "prevalência de sedentarismo" se os critérios de análise são grosseiramente díspares. Em que pese a existência de questionários validados que poderiam tentar aproximar os resultados, o que seria uma criança sedentária? Crianças, principalmente em localidades carentes, muito raramente, não se movimentam durante seu dia-a-dia, seja realizando brincadeiras infantis ou se deslocando da casa para a escola e desta para casa, uma vez que os meios de transporte são, muitas vezes, precários.

Assim, pressuponho que estas classificações estejam muito mais movidas por critérios ideológicos do que científicos e ao criarem noções normativas parecem destinadas a ideais persecutórios.

Alexandre Palma RIO DE JANeiro - RJ

Referências

I. Nietzsche FW. A gaia ciência. Rio de Janeiro: Companhia das Letras; 200 I . 2. Saguy AC, Riley KW. Weighing both sides: morality, mortality, and framing contests over obesity. J Health Polit Policy Law. 2005;30(5):869-921 .

3. Lupton D. The imperative of health: public health and the regulated body. London: Sage; 1995.

4. Paul Campos P, Saguy A, Ernsberger P, Oliver E, Gaesser G. The epidemiology of overweight and obesity: public health crisis or moral panic? Int J Epidemiol. 2006;35(I):55-60.

5. Gard M, Wright J. The obesity epidemic: science, morality and ideology. London: Routledge; 2005

6. Barreto ACNG, Brasil LMP, Maranhão HS. Sobrepeso: uma nova realidade no estado nutricional de pré-escolares de Natal, RN. Rev Assoc Med Bras. 2007;53(4):3 I I-6.

7. Escobar AMU, Valente MH. Sobrepeso: uma nova realidade no estado nutricional de pré-escolares de Natal, RN. Rev Assoc Med Bras. 2007;53(5):378-9.

\section{Resposta dos autores}

Segundo Nietzsche, em 1882: "Muitas vezes consideramos uma idéia mais verdadeira apenas porque há qualquer coisa de muito belo e divino no ritmo e na forma métrica do seu enunciado. Não é divertido notar que os filósofos mais sérios, por mais rigorosos que sejam na sua busca da certeza, citam freqüentemente as palavras dos poetas para dar às suas idéias mais força e credibilidade? E, no entanto, é mais perigoso para uma verdade se um poeta concorda com ela do que se ele a contradiz! Porque, como dizia Homero, "Muitas mentiras contam os poetas"'. Utilizamos texto também retirado da "A Gaia Ciência"' para demonstrar a importância das discussões e questionamentos ao fazermos pesquisa científica, lembrando que as críticas levam ao aperfeiçoamento e ao crescimento da ciência. Devemos, no entanto, considerar alguns aspectos importantes:

I. A obesidade é tida como problema mundial de saúde pública pela Organização Mundial de Saúde². Vários estudos demonstram a relação existente entre essa enfermidade e várias co-morbidades como hipertensão arterial, Diabetes mellitus 
tipo 2, síndrome metabólica, problemas ortopédicos, doenças respiratórias, acidente vascular cerebral, doenças coronarianas, problemas psicológicos, alterações cutâneas, enfermidades gastrointestinais, doenças neoplásicas, diminuição da autoestima e outras. Lima et al, em 2004, ${ }^{3}$ encontraram elevada peroxidação de lipídeos no plasma de crianças obesas, sendo esse um importante fator de risco para o desenvolvimento de complicações nestes pacientes. A Federação Internacional de Diabetes considera a obesidade como o principal fator de risco modificável de desenvolver Diabetes mellitus tipo $2^{4}$. Segundo - Relatório sobre Saúde no Mundo da Organização Panamericana de Saúde (2002), aproximadamente 58\% dos casos de diabetes, $21 \%$ de cardiopatias isquêmicas e $8 \%-42 \%$ de certos tipos de câncer estão relacionados com a presença de índice de massa corpórea superiores a $21 \mathrm{~kg} / \mathrm{m}^{2}$ em adultos ${ }^{5}$. Freedman et al. (2003), ${ }^{6}$ relataram o efeito adverso cumulativo presente em indivíduos com obesidade na infância, que assim permaneceram na vida adulta. Foram encontrados nesses pacientes os mais altos níveis de aumento da espessura da camada íntima da artéria carótida, sendo esse um indicador de aterosclerose generalizada. Esse achado não foi encontrado em adultos obesos que tiveram peso normal quando crianças. Podemos citar várias outras referências, baseadas em estudos de evidência classe 2 e 3 que demonstram a associação do excesso de peso com as morbidades acima. $\bigcirc$ estudo de Campos et al. ${ }^{7}$, citado pelo colega, porém, não tem evidências científicas suficientes para suportar a hipótese que a obesidade não é maléfica para a saúde. Algumas de suas conclusões são que algumas das comorbidades citadas acima estão associadas com o excesso de gordura no organismo e existe a necessidade de mudança da dieta e aumento na atividade física. Conclusões essas idênticas às que chegaram os estudiosos em obesidade. É óbvio que relacionar simplesmente obesidade a aumento na morbimortalidade é estar simplificando todo o processo fisiopatológico a apenas uma relação causa-efeito. Nesse estudo, também não há a citação de nenhum artigo que comprove ser o excesso de gordura benéfico para o organismo, ou seja, que contrariem indubitavelmente os trabalhos anteriormente realizados, demonstradores do prejuízo do acúmulo excessivo de gordura corporal.

2. Não foi objetivo do nosso artigo $^{8}$ discutir sobre as medidas da obesidade na infância. Reconhecemos a fragilidade das medidas atualmente utilizadas para verificar adiposidade e sabemos da necessidade de relacionar essas medidas com morbimortalidade e implicações clínicas. Mas, ressaltamos as dificuldades, inclusive do ponto de vista ético, em realizar estudos em crianças para avaliar esses aspectos. Há trabalhos mostrando que o índice de massa corpórea (IMC) é o melhor indicador de obesidade entre crianças, comparando com outros índices de peso/estatura, como o índice de Rohrer e o índice de Benn. ${ }^{9}$ A desvantagem é que nenhum desses índices distingue o excesso de peso devido ao aumento de tecido gorduroso do aumento relacionado à musculatura, tecido ósseo ou edema. Entendemos que o IMC em percentil com pontos de corte recomendados pelo CDC/Atlanta possui várias restrições, entre elas o fato de as curvas terem sido construídas apenas por população norte-americana, limitando sua aplicabilidade internacional, além de utilizarem pontos de corte que não comprovam relação com o desfecho clínico. Não obstante, esse critério foi utilizado porque grande parte dos estudos anteriores utiliza essas curvas, o que facilitaria a comparação de resultados. É importante também ressaltar que várias pesquisas demonstraram boa correlação do IMC com outras medidas de adiposidade, como a espessura da prega tricipital e circunferência abdominal. Esse índice também tem a vantagem de ser um método fácil e prático para ser aplicado em programas epidemiológicos de triagem de excesso de peso com tamanho de amostra grande como o do nosso estudo (372I crianças). Sabemos que são estudos iniciais e de triagem e, é óbvio, que as crianças assim diagnosticadas com excesso de peso deverão passar por avaliações mais rigorosas, com a realização de métodos mais específicos para o diagnóstico de obesidade e que não tem aplicabilidade em grandes populações como método de triagem.

3. Em relação à comparação com estudos anteriores realizados no Brasil que utilizaram outros critérios diagnósticos ( $Z$ escore do índice peso/estatura), nós tivemos o cuidado de realizar um estudo em que foi feita a comparação dos dois métodos (cujos resultados foram apresentados em evento internacional e enviados para publicação). Nessa avaliação, considerando o $Z$ escore como método padrão (até então recomendado pela OMS), encontramos que o IMC em percentil teve sensibilidade e especificidade de 97,7\% e 96,9\%, respectivamente, valor preditivo positivo de $91,2 \%$ e negativo de 99,2\%, com razão de verossimilhança de 31,5 para o diagnóstico de excesso de peso. Esses resultados indicam que os dois métodos possam ser comparados. Infelizmente, os únicos estudos com amostra representativa da população brasileira tomaram como critério diagnóstico o $Z$ escore do índice peso/estatura. Não utilizamos esse método devido ao fato de o IMC ser bem mais abrangente e recomendado por órgãos internacionais que estudam obesidade, como o IOTF assim como a OMS.

4. Com relação ao questionamento sobre sedentarismo, também não foi objetivo do nosso estudo discutir suas definições e critérios de análise, tampouco sua avaliação. No entanto, não foi possível ignorar a publicação do autor dessa correspondência e seus colegas, em $200 I^{10}$, de artigo em que analisaram pequena amostra da população que foi escolhida de forma não aleatória e utilizaram definições de sobrepeso não reconhecidas pelas principais organizações e sociedades de estudo da obesidade. Além disso, empregarem a definição de sedentarismo baseada em horas gastas com televisão e atividade física, apesar de seu comentário ao nosso trabalho fazer referência aos 
critérios de análise de sedentarismo "grosseiramente díspares" e indagar se existiria questionários válidos para a definição do que seria uma criança sedentária, desmerecendo, portanto, seu próprio artigo. Interessante ler a conclusão deste manuscrito, que também defende a idéia da necessidade de prevenção da obesidade na infância e adolescência. Os pontos de vistas levantados então foram bastante semelhantes aos que foram por nós utilizados, o que nos leva a crer que o prezado colega, na verdade, contradiz e critica seu próprio trabalho.

5. Idéias persecutórias, segundo a psiquiatria, são aquelas em que a pessoa acredita estar sendo atormentada, seguida, enganada, espionada ou ridicularizada e faz parte de quadros clínicos de distúrbios psicóticos. Acreditamos que o colega tenha empregado mal essa terminologia ou baseou-se em opiniões pessoais que não é oportuno nem adequado expor em nível científico.

6. Lembramos ainda que nosso artigo não teve nenhum conflito de interesses.

Por fim, agradecemos às críticas realizadas e a oportunidade de esclarecê-las e assim enriquecer a nossa discussão. Na oportunidade, renovamos nossa disposição para quaisquer outros esclarecimentos.
Referências

I. Nietzsche FW. A Gaia Ciência. Rio de Janeiro: Ediouro; s/d.

2. Obesity: preventing and managing the global epidemic. Report of a WHO consultation. World Health Organ Tech Rep Ser. 2000;894: I-253.

3. Lima SC, Arrais RF, Almeida MG, Souza ZM, Pedrosa LF. Perfil lipídico e peroxidação de lipídeos no plasma em crianças e adolescentes com sobrepeso e obesidade. J Pediatr (Rio J). 2004;80( I):23-8.

4. Internacional Diabetes Federation. Dia Mundial de Diabetes 2004. Combate la obesidad prevén la diabetes. Hoja de datos sobre la obesidad [citado 2004 nov 14]. Disponível em: http://www.idf.org/home/index.cfm?unode=704F5B9C3C6F-4CEE-B7-CI 5ECAA4BBD4.

5. OPAS. Obesidade e Excesso de peso. In: Organização Panamericana de Saúde. Doenças crônico-degenerativas e obesidade: estratégia mundial sobre alimentação saudável, atividade física e saúde. Brasília: Organização Panamericana da Saúde; 2003. p.27-34.

6. Freedman DS, Dietz WH, Tang R, Mensah GA, Bond MG, Urbina EM, et al. The relation of obesity throught life to carotid intima-media thickness in adulthood: The Bogalusa Heart Study. Int J Obes Relat Metab Disord. 2004;28(I): I 59-66

7. Campos P, Saguy A, Ernsberger P, Oliver E, Gaesser G. The epidemiology of overweight and obesity: public health crisis or moral panic?. Int J Epidemiol. 2006;35:55-60

8. Barreto ACNG, Brasil LMP, Maranhão HS. Sobrepeso: uma nova realidade no estado nutricional de pré-escolares de Natal, RN. Rev Assoc Med Bras. 2007;53(4):31 I-6.

9. Mei Z, Grummer-Strawn LM, Pietrobelli A, Goulding A, Goran MI, Dietz WH. Validity of body mass index compared with other body-composition screening indexes for the assessment of body fatness in children and adolescents. Am J Clin Nutr. 2002 Jun;75(6):978-85.

10. Pimenta APAA, Palma A. Perfil epidemiológico da obesidade em crianças: relação entre televisão, atividade física e obesidade. Rev Bras Cien Mov. $2001 ; 9(4): 19-24$. 